\title{
4D printing of shape memory polylactic acid (PLA) components: Investigating the role of the operational parameters in fused deposition modelling (FDM)
}

\author{
M. Barletta ${ }^{\mathrm{a}, *}$, A. Gisario ${ }^{\mathrm{b}}$, M. Mehrpouya ${ }^{\mathrm{a}, \mathrm{c}}$ \\ ${ }^{a}$ Università degli Studi Roma Tre, Dipartimento di Ingegneria, Via Vito Volterra 62, 00146 Roma, Italy \\ ${ }^{\mathrm{b}}$ Sapienza Università degli Studi di Roma, Dipartimento di Ingegneria Meccanica e Aerospaziale, Via Eudossiana 18, 00184 Roma, Italy \\ ${ }^{\mathrm{c}}$ Faculty of Engineering Technology, University of Twente, P.O. Box 217, 7500 AE Enschede, the Netherlands
}

\section{A R T I C L E I N F O}

\section{Keywords:}

4D printing

Fused deposition modelling (FDM)

Shape memory polymers (SMPs)

Polylactic acid (PLA)

Design for manufacturing

\begin{abstract}
A B S T R A C T
Shape Memory Polymers (SMPs) are attracting considerable interest both in the scientific and industrial fields. Among them, polylactic acid (PLA) is particularly appreciated, as it is a bioderivation polymer, biodegradable and compostable with high thicknesses, with excellent shape memory properties, which can be activated by varying the temperature of the material across the glass transition temperature. In this study, PLA is used in the 4D printing process for the manufacturing of complex geometry components by Fused Deposition Modelling (FDM), with a potential as stress-absorbers. The shape memory properties of the manufactured component have been tested varying the operational parameters of the printing process (i.e., the temperature at the nozzle, the deposition speed, the layer thickness) as well as the activation temperature that allows the recovery of the initial shape. In particular, the time needed to recover the shape and the percentage of recovery with respect to the initial shape were assessed. Experimental results have shown that PLA components have adequate shape memory properties, with short recovery times and high recovery rates being easily achievable. Among process parameters, the activation temperature is found to be the most significant parameter to trigger the recovery of the initial shape in the shortest possible time.
\end{abstract}

\section{Introduction}

3D printing processes have become increasingly important since they were conceived in the 1980s. 3D printing technologies can be successfully applied in numerous market segments: production of components with complex or custom geometry [1,2], design of new products [3], biotechnology and life sciences $[4,5]$, leisure and free time [6,7]. There are numerous technologies that allow to implement the 3D printing process [8]. The most commonly adopted techniques include: (i) Fused Filament Fabrication (FFF) or Fused Deposition Modelling (FDM), (ii) Digital Light Processing (DLP), (iii) Color Jet (CJP) and Multi Jet Printing (MJP); (iv) Stereolithography (SLA); (v) Selective Laser Sintering (SLS); (vi) Direct Metal Printing (DMP). The devices that allow to implement the 3D printing process are introduced on the market at increasingly competitive costs, allowing access to the technology to an ever-wider audience. The expansion of users allows an increase in exchanges between professionals, with considerable advantage for the refinement of design and operational techniques. In the current state of the art, the $3 \mathrm{D}$ printing techniques, while presenting various advantages such as rapid times and reduced costs for the prototyping of a new product, only allow the production of static components that do not have the ability to modify their shape through the application, for example, of an external stimulus or the introduction of a kinematic mechanism [9]. Therefore, the production of assemblies often requires the use of support structures in 3D printing, which, in fact, reduce or cancel the process advantages. Furthermore, 3D printing techniques are intrinsically limited in terms of the size of the finished products, as well as on the size of the economic production batch which must necessarily be small [10].

More recently, by combining additive manufacturing technologies with the development of intelligent materials, it is possible to pursue the production of new 3D printable products that can, for example, change the shape or the volume when exposed to different stimuli, allowing to obtain so-called 4D objects [11]. These objects possess, as such,

\footnotetext{
* Corresponding author.

E-mail address: massimiliano.barletta@uniroma3.it (M. Barletta).
} 
considerable application potential. For instance, SMPs can dynamically change their shape by being exposed to different stimuli $[12,13,14]$. SMPs are often 3D printed by FDM [15]. The use of SMPs involves, as a first stage, the setting of a starting form. Subsequently, they can be fixed in a temporary form through the application of a permanent deformation by an external force. This second stage is called programming. After being programmed, SMPs can return to their permanent form by applying an external stimulus (third stage). The process can possibly be iterated. SMPs can generally be programmed using thermomechanical stimuli. SMPs are inexpensive, have low density, extensive deformability in the elastic field. In some cases, moreover, SMPs can be biodegradable, as well as be sensitive to numerous stimuli [16]. To implement the transition between the various stages, it is not necessary to use complex electronic actuators. SMPs are often manufactured by 3D printing processes, thus giving rise to additive manufacturing technologies called 4D printing. Of interest, polylactic acid (PLA) is a biodegradable SMP, susceptible to thermal stimuli. Some studies are available that employ PLA as SMP [17], often in mixture with thermoplastic elastomers [18,10]. PLA-based SMPs [19,20] can be programmed by applying an external stress when their temperature is higher than the glass transition temperature. They can be locked in the new state by bringing the deformed material below the glass transition temperature. Their shape can be restored by re-heating them to a temperature higher than the glass transition temperature.

Although the studies concerning the use of PLA as SMP are quite numerous, there is still not enough data on the link between the parameters of the $3 \mathrm{D}$ printing process and the establishment of shape memory properties of the items themselves. Furthermore, there are not enough data that correlate the shape memory properties to the temperature used to carry out the stimulus. In this framework, the present study deals with the applications of PLA in the 4D printing process for the manufacturing of a cellular structure using Fused Deposition Modeling (FDM). Shape memory of the samples was investigated by varying the nozzle temperature of the printer extruder, the deposition speed of the printing layer, the layer thickness. In addition, the activation temperature during the recovery tests of the sample was also investigated. In particular, the recovery time and percentage of the samples were evaluated by varying the $3 \mathrm{D}$ printing process parameters and the so-called activation temperature. Experimental findings show the PLA samples have remarkable shape memory capabilities, with rather short recovery times and high recovery percentage. The activation temperature is found to be the most significant parameter to restore the initial form of the samples in the shortest possible time range.

\section{Materials and methods}

Materials

Filaments in PLLA, $\sim 96 \%$ L-Isomer content, $\sim 1.25 \mathrm{kDa}$ molecular weight, $\sim 1.6$ polydispersity index, (Alfaplus, Filoalfa, Ozzero (MI), Italy) with a diameter of $1.75 \pm 0.05 \mathrm{~mm}$ were used during the experimental tests [21]. The PLLA is modified by the addition of less than 10 $\%$ of an elastomeric material (TPU, thermoplastic polyurethane). The thermo-physical and mechanical properties of the material are reported

Table 1

Properties of PLA filaments ( ${ }^{*}$ DSC $=$ Differential Scanning Calorimeter).

\begin{tabular}{lll}
\hline Properties & Value & Regulation \\
\hline Density & $124 \mathrm{~g} / \mathrm{cm}^{3}$ & ISO 1183 \\
Melting Temperature & $177^{\circ} \mathrm{C}$ & DSC $^{*}$ \\
Glass Transition Temperature & $57{ }^{\circ} \mathrm{C}$ & DSC* \\
Charpy & $25 \mathrm{~kJ} / \mathrm{m}^{2}$ & ISO $179-1 \mathrm{eU}$ \\
Elongation at break at $20^{\circ} \mathrm{C}$ & $4,1 \%$ & ISO 527 \\
Tensile strength at break at $20^{\circ} \mathrm{C}$ & $43 \mathrm{MPa}$ & ISO 527 \\
Flexure Modulus at $20^{\circ} \mathrm{C}$ & $3070 \mathrm{MPa}$ & ISO 527 \\
Heat Deflection Temperature & $58^{\circ} \mathrm{C}$ & ISO 75 \\
\hline
\end{tabular}

in Table 1.

\section{Design of the components}

The need to create lightweight structures with high stiffness has led to the development of sandwich-type structures in recent years. They consist of two components: the rigid and external skin, and the core, mostly consisting of hollow volume. Among the advantages offered by structures of this type, there is the possibility of composing multiple materials with different characteristics. In order to create skins and cores of different materials, it is possible to fill the cavities of the core with light materials such as foams (for example, flame retardant foams, insulating foams or capable of resisting the energy of an impact). As a result of the light weight of the resulting components, the first and most frequent uses of sandwich structures are in the aerospace field or as stress-absorbers in many technological fields. The choice of the core texture allows to obtain multiple mechanical properties. A first distinction can be made between in-plane and out-of-plane structures $[22,23]$ : the core is the same but the arrangement of the structure changes, with the in-plane type being of particular interest for the application in which a high stiffness, especially for applications related to the structure impact resistance, plays a crucial role. In this work, on-plane structures will be, however, considered. Fig. 1 reports the geometry of the sample. The size is approximately $30 \times 30 \times 30 \mathrm{~mm}^{3}$. The model of the component has been previously made at CAD. Subsequently, the file was converted to '.stl' format. Finally, the '.gcode' file was created using the software Ultimate Cura 3.2.1 to be supplied as input to the $3 \mathrm{D}$ printer for the manufacturing of the part.

\section{Equipments}

The manufacturing of the samples was performed using an openchamber 3D printing (Meeds, Zaturi, Rome, Italy). The system, based on FDM technology, is equipped with an aluminum frame, an extruder with a diameter of $0.4 \mathrm{~mm}$ and a glass-built heated plate. The filament is located on top of the aluminum space frame. The maximum printing volume is $200 \mathrm{~mm}$ in diameter and $300 \mathrm{~mm}$ in height. The maximum temperature at the nozzle is $260^{\circ} \mathrm{C}$. The maximum temperature of the built-in heated plate is $110{ }^{\circ} \mathrm{C}$. The layer resolution is $100 \mu \mathrm{m}$. The use of the heated built-in plate is necessary to guarantee the adhesion of the model during the first steps. The first layers are of fundamental importance, as their misalignment can cause a propagation of the defect throughout the vertical extension of the sample.

\section{Testing procedure}

The experimental test consists of two different stages. In the first, the compression tests of the samples are performed in order to program the new shapes of the samples. Programming of the samples was performed at ambient temperature. During programming, the deformation speed of the samples was set at $1 \mathrm{~mm} / \mathrm{min}$ in order to achieve the final shape very slowly, thus allowing the samples to gradually adapt to the new shape. Additionally, the mechanical response of the samples by varying the printing operating parameters is investigated. In the second, the capacity of the samples to recover the initial shape varying the printing operating parameters and the activation temperature to trigger the recovery process was investigated. The experimental procedure can, therefore, be divided into the following operations:

- Identification of a set of printing parameters (Table 2) and manufacturing of the samples;

- Performing the compression tests on the samples to program them and assess the mechanical response varying the printing operating parameters and data analysis; 


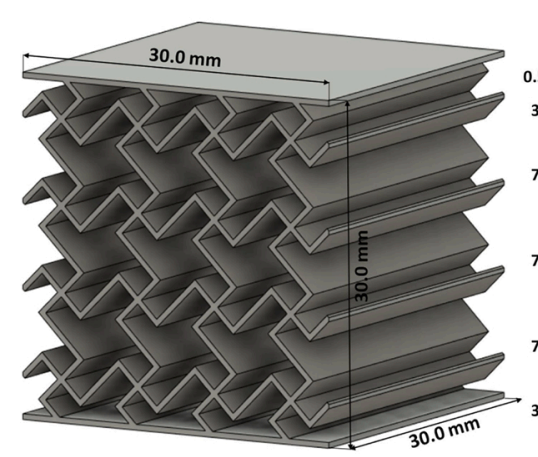

(a)

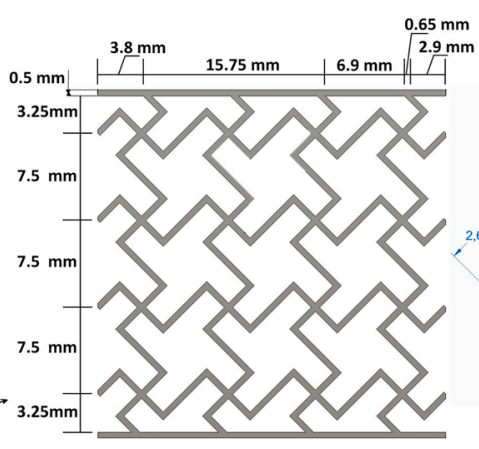

(b)

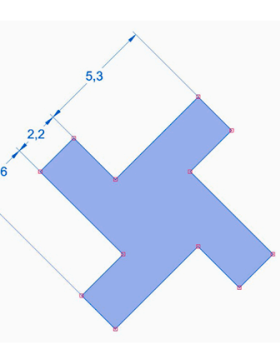

(c)

Fig. 1. Model of the component.

Table 2

Experimental schedule.

\begin{tabular}{lll}
\hline Nozzle temperature, ${ }^{\circ} \mathrm{C}$ & Printing Speed, $\mathrm{mm} / \mathrm{s}$ & Layer thickness, $\mathrm{mm}$ \\
\hline $180^{\circ} \mathrm{C}$ & $40 \mathrm{~mm} / \mathrm{s}$ & $0.15 \mathrm{~mm}$ \\
$210^{\circ} \mathrm{C}$ & $80 \mathrm{~mm} / \mathrm{s}$ & $0.30 \mathrm{~mm}$ \\
\hline
\end{tabular}

- Immersion of the sample in a temperature-adjustable water tank to recover the initial shape at post heating temperature of 65,75 and $85^{\circ} \mathrm{C}$ (higher than the glass transition temperature of the PLA);

- Analysis of the shape recovery for the samples manufactured varying the printing operating parameters and data processing.

Recovery time is defined in Fig. 2. The starting height of each sample is $30 \mathrm{~mm}$. After compression tests (deformation imposed of $8 \mathrm{~mm}$, final height $30-8=22 \mathrm{~mm}$ ), the maximum height that can be restored on the samples after full recovery is $29.6 \mathrm{~mm}$. All the tested samples, after programming and recovering steps, approach the final height of about $29.6 \mathrm{~mm}$ or slightly less (96-99\% of the initial height). The main difference resides in the time needed by the different samples to get the full restore of the shape. This is therefore the recovery time, that is the time needed by each sample to recover the most. This is found to be dependent on the temperature set during the recovery tests. As shown in Fig. 2, the time needed by the samples to restore the shape the most is lower at any time the temperature set during the recovery tests is increased. At the highest temperature of $85{ }^{\circ} \mathrm{C}$, the recovery time is very quick and it is regulated by the physical opposition (the drag resistance) of the hot fluid in which the sample is dipped during the shape recovery tests. Recovery percentage is, on the other side, defined as the ratio of the current height

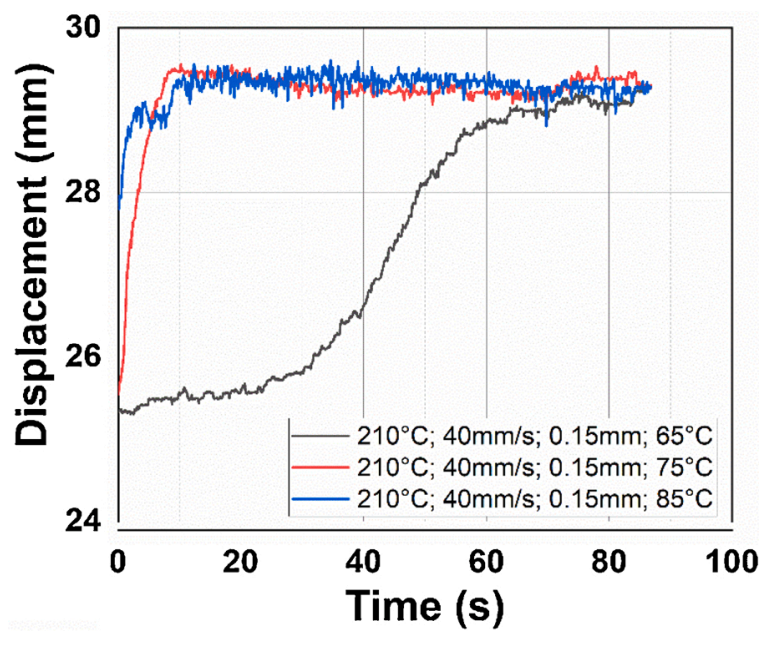

Fig. 2. Definition of recovery percentage. of the samples after full recovery in the hot-water tank to the maximum height the samples can approach after the full recovery following the programming step (that is, $29.6 \mathrm{~mm}$ ).

All the experimental conditions were replicated three times in order to ensure reliability and repeatability of the experimental results. Main effects and interaction plots were used to report the experimental results. The Main Effect Plots (MEP) are the graphs plotting the means for each value of a categorical variable. This is used to determine whether or not the main effect is present for the categorical variable. If the line is horizontal, in other words, parallel to the x-axis, then there is no main effect exists. Interaction plot (IP) is used to show the relationship between one categorical factor and a continuous response depends on the value of the second categorical factor. This plot displays means for the levels of one factor on the $\mathrm{x}$-axis and a separate line for each level of another factor. If lines in the plot run parallel, no interaction occurs. If the lines run not parallel, an interaction occurs. The more non parallel the lines are, the greater the strength of the interaction.

\section{Characterization}

The compression tests were carried out with a universal dynamometer (Instron 3367, Instron, Norwood, Massachusetts, United States) for static and dynamic tests in both tensile and compression mode. The device acquires the data relating to the movement as the applied force changes. Experimental data are transferred to a software for storage. The maximum deformation at the end of the test was kept fixed at $8 \mathrm{~mm}$. The deformation speed was set at $1 \mathrm{~mm} / \mathrm{min}$. The heat necessary to reach the activation temperature of the PLA component was provided by a temperature-controlled tank (Corio C series, Julabo GmbH, Seelbach, Germany). The temperature of the $19 \mathrm{~L}$ tank, made in transparent polycarbonate, is regulated by a temperature controller with a resolution of $0.01{ }^{\circ} \mathrm{C}$ and stability of the temperature of $0.03^{\circ} \mathrm{C}$. To quantify the shape recovery on a comparative base, a video tracking software, tracker video analysis and modeling tool were used. The system analyzes the frames of the video and determines the movement of a tracer point, after the setting of the coordinated axes and a geometric reference. A full HD camcorder (GZ-E205, JVC, Yokohama, Kanagawa, Japan) was used for the specific purpose.

\section{Results and discussion}

Programming the SMPs

Programming the SMPs was performed by a compression test. The samples were submitted to a very slow deformation at constant speed $(1 \mathrm{~mm} / \mathrm{min})$ in a dynamometer at ambient temperature. Maximum deformation was kept constant at $8 \mathrm{~mm}$, so that the final structure height after programming was always set at $22 \mathrm{~mm}$ (30 mm starting height of the sample minus $8 \mathrm{~mm}$ deformation). Fig. 3 shows the trends 

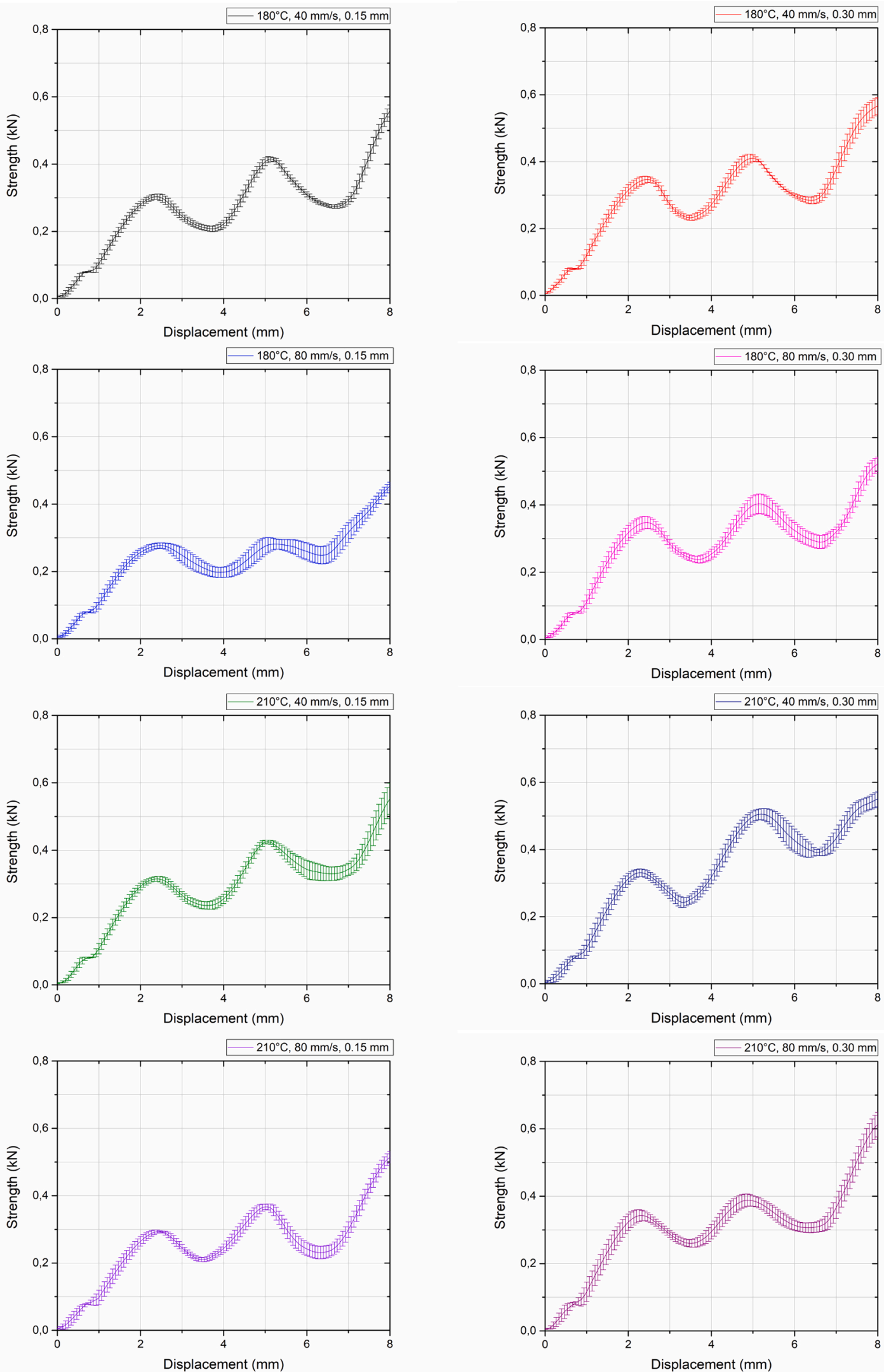

Fig. 3. Compression strength vs. displacement varying nozzle temperature, printing speed and layer thickness. 
of compression load vs. displacement for the samples manufactured by varying the nozzle temperature, the printing speed and the layer thickness. The trends of the compressive load show three subsequent peaks, which correspond to the collapse of the three layers of the cellular structures of which each individual sample is composed. The analysis of the second peaks is reported in Figs. 4 and 5. Fig. 4 reports the main effect plot of the compressive strength measured at the second peak of the compressive strength in Fig. 3. It is possible to observe the increase in compressive load at the second peak increasing the nozzle temperature from 180 to $210{ }^{\circ} \mathrm{C}$ and the layer thickness from 0.15 to $0.30 \mathrm{~mm}$, respectively. In addition, it is also possible to observe the decreasing of the compressive strength at the second peak by increasing the printing speed from 40 to $80 \mathrm{~mm} / \mathrm{s}$.

PLA features a melting peak of $\sim 177^{\circ} \mathrm{C}$. Printing the PLA at a temperature of $180^{\circ} \mathrm{C}$ means operating in conditions very close to the melting temperature of the material. At $180^{\circ} \mathrm{C}$, the material is characterized by a very low viscosity (that is, a very low melt flow rate or a very high molecular weight), which makes its formability rather complex during the printing operation [24]. The extruder cannot reach adequate working pressure at the nozzle and the printed material is consequently decidedly less homogeneous and compact. Increasing the printing speed produces a similar effect. The extruder is unable to reach enough pressure at the nozzle, as the flow rate of material it must guarantee is excessively high. Even in this case, the printed material is less homogeneous and compact [25]. In both cases, the lower level of homogeneity and compactness achieved by the printed material is associated with a decrease in the pressure value measured at the second peak, necessary to deform the sample, as visible in the Main Effects Plot in Fig. 4. An increase in the thickness of the layer deposited during the printing process causes an increase in the overall stiffness of the structure and, therefore, an increase in the compression load value measured at the second peak, as shown in the Main Effects Plot in Fig. 4.

Fig. 5 shows the Interactions Plot for the compression load. All the trends run parallel, being this a sign of lack of interactions among the different experimental factors investigated.

Fig. 6 reports the overall deformation of the sample structure after the programming step. However, the sample does not show any significant damage, like cracks or fractures as a result of the very low deformation speed set during programming. At the end of the programming step, the overall height of the sample is set, as specified before, at $22 \mathrm{~mm}$.

Fig. 7 reports the evolution of the elastic recovery of the samples varying the operating parameters. The increase in the elastic recovery, when the nozzle temperature is set at $210{ }^{\circ} \mathrm{C}$, is decidedly ascribable to the better homogeneity and compactness of the PLA material.

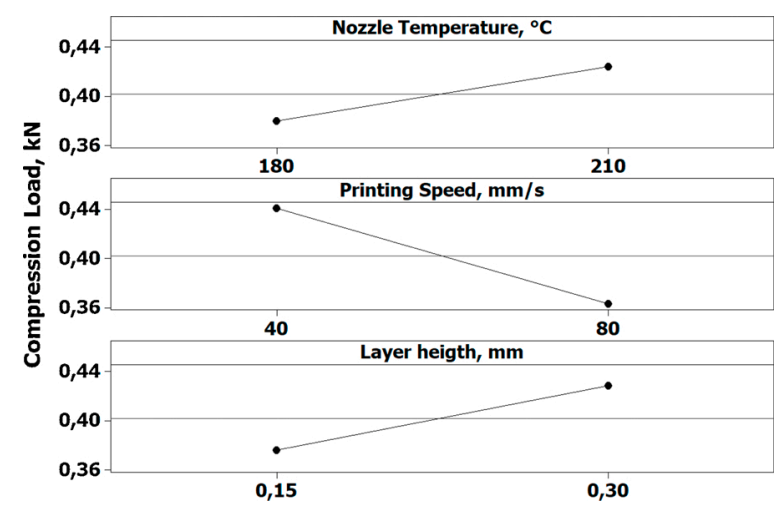

Fig. 4. Main effects plot of the compression strength measured at the second peak.

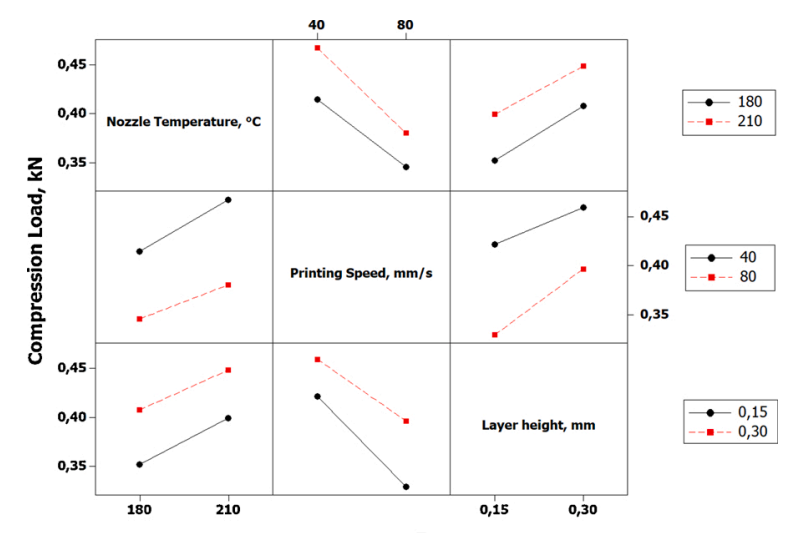

Fig. 5. Interactions plot of the compression strength measured at the second peak.

\section{Actuating the SMPs}

Actuation of the SMPs was performed by post-heating the samples inside a water bath at temperature of 65,75 and $85^{\circ} \mathrm{C}$. The temperatures are set at values that are always higher than the glass transition temperature of the PLA $\left(\sim 57^{\circ} \mathrm{C}\right)$. Fig. 8 shows the different steps through which the PLA model obtained by 3D printing recovers its initial shape, exhibiting its remarkable shape memory properties. Fig. 8a shows the sample immediately after the programming phase. The initial shape depends not only on the maximum deformation set during the compression step, but also on the elastic recovery the individual model exhibits, which is a function of process conditions during $3 \mathrm{D}$ printing as shown in Fig. 7. Fig. 8b and c show the intermediate steps of the recovery phase, with the model that is restoring its starting geometry. Note the individual and progressive recovery of the individual cells of the model, which were initially crushed, to open-up and regenerate almost completely. In Fig. 8d, the complete regeneration of the cellular structure can be noticed, with the cells having completely opened. At the end of the recovery process, the model almost assumed the form it had before the programming phase (that is, before compression), exhibiting excellent shape memory capabilities in agreement with [26,27]. In Fig. 7, the trends of the elastic recovery of the models are shown as the process conditions vary, after the programming phase. Basically, all models underwent a deformation of $8 \mathrm{~mm}$, bringing their height from the initial value of $30 \mathrm{~mm}$ to $22 \mathrm{~mm}$, this being dependent by the setting of programming step. However, when the compression load is released, the samples tend to recover slightly their shapes in the elastic field, as shown in Fig. 7. In particular, the greatest recovery percentage is found by those samples that have been processed at nozzle temperature of $210^{\circ} \mathrm{C}$. As said, the nozzle temperature allows to establish the right pressure of the polymer melt at the extruder nozzle during the 3D printing process [24]. For this reason, the material structure of the models produced setting the nozzle temperature at $210{ }^{\circ} \mathrm{C}$ is decidedly more homogeneous and compact, exhibiting a more ductile behavior and, therefore, a greater elastic recovery already at room temperature, that is, immediately after the programming phase.

Figs. 9 and 10 reports the main effects plot of the recovery time and of the recovery percentage varying the operating parameters under investigation, that is, the settings of the printing device (nozzle temperature, printing velocity and layer height), and the post-heating temperature at which the models are annealed to activate the shape memory properties.

Similarly, Fig. 11 and 12 report the interactions plot of the recovery time and of the recovery percentage varying the same operating parameters. Figs. 9 and 10 shows, in particular, the $\delta$-values of the recovery time and percentage. The changes in post-heating temperature cause the highest $\delta$-values of the recovery time and percentage. Increasing the 


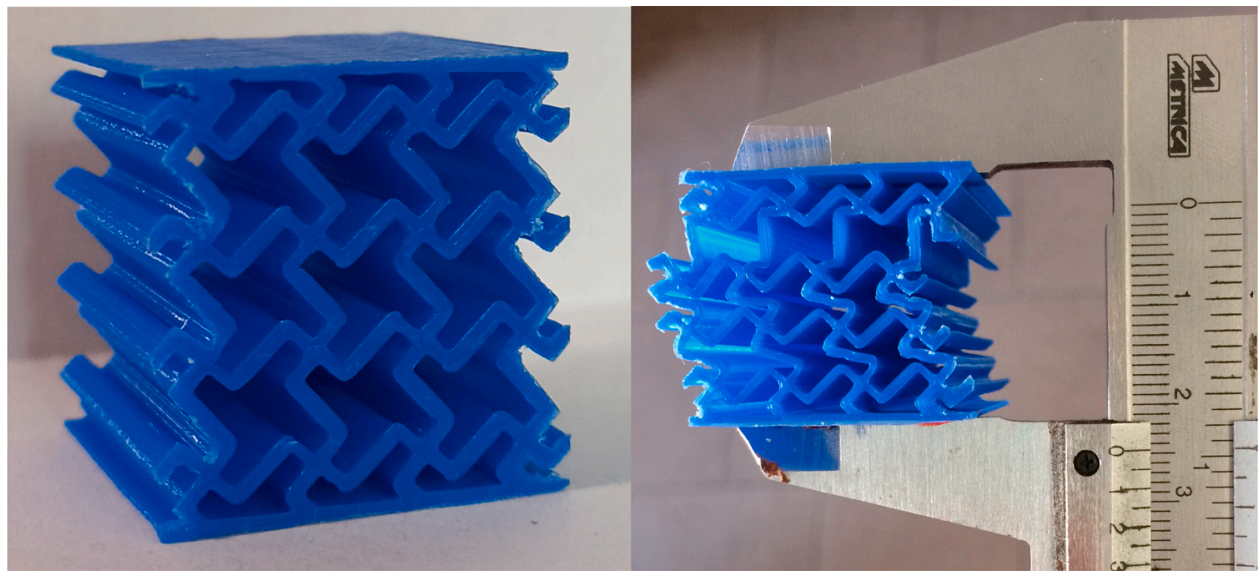

Fig. 6. Image of the sample before and after the compression test (after programming step).

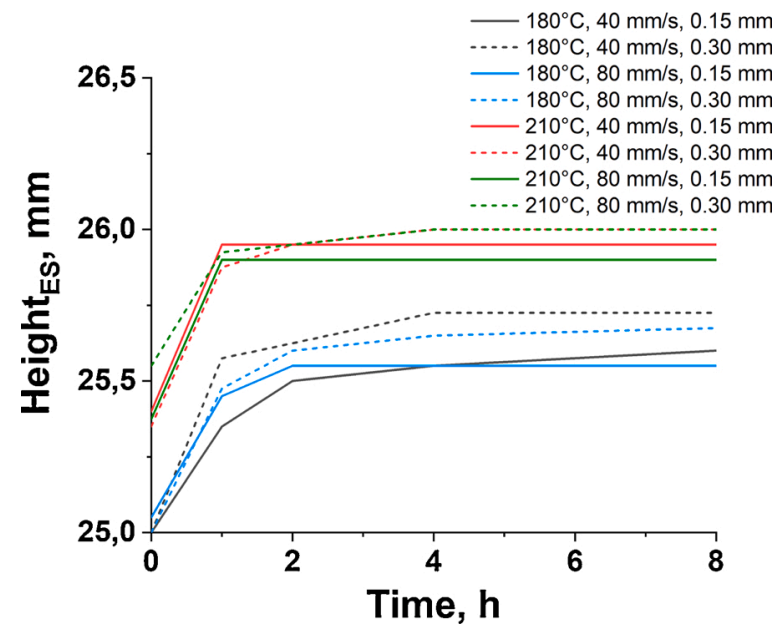

Fig. 7. Trend of the elastic recovery of the samples manufactured varying the different operating parameters.

post-heating temperature speeds up the recovery of the PLA and, also, the recovery extent with respect to the initial shape of the sample. Actuation of PLA happens, notoriously, at temperature higher than the glass transition. When the post-heating temperature is increased, the time the sample takes to get at temperature above the glass transition (thermal inertia) is shorter. In fact, the sample can reach the activation threshold (that is, the glass transition temperature) much faster. For this reason, the sample recovers faster and restores almost completely the initial shape. Recovery time as low as $10 \mathrm{~s}$ can be achieved by setting the post-heating temperature of the samples in the water bath at $85^{\circ} \mathrm{C}$. Similarly, recovery percentage as much high as $99.3 \%$ can be reached, when the post-heating temperature is set at $85{ }^{\circ} \mathrm{C}$. These results state the effectiveness of the PLA material and of the sample geometry. The recovery percentage achieved are decidedly higher if compared with

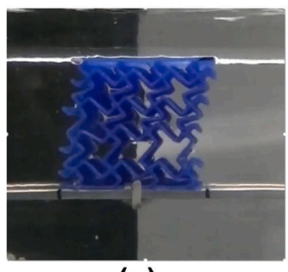

(a)

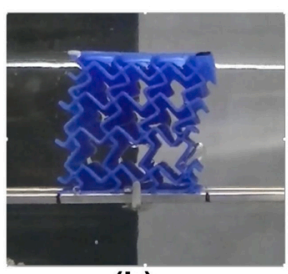

(b)

typical data reported in the literature for similar systems [28] or fully biodegradable PCL/PLA blends [29]. The built model boasts excellent shape memory properties. An increase in nozzle temperature and in layer height favors the establishment of higher recovery percentage, although longer time is necessary to restore the initial shape of the samples. This is ascribed to the most favorable process conditions that can be achieved using higher nozzle temperature and layer height. Under these circumstances, the PLA is sufficiently fluid to be processed adequately by the printing equipment. In fact, at temperature of $210^{\circ} \mathrm{C}$, the MFR of the PLA decreases and the extruder can reach an adequate melt pressure at the nozzle, allowing the manufacturing of a sample characterized by a better homogeneity and compactness. Higher nozzle temperatures are excluded, as they would lead to polymer thermal degradation. The increase in the layer height favors, instead, the homogeneity and the compactness of the printed material, thus promoting a better recovery of the samples to their initial shapes. An increase in printing speed both decreases the recovery time and percentage. When the printing speed is increased, the melt pressure at the extruder nozzle

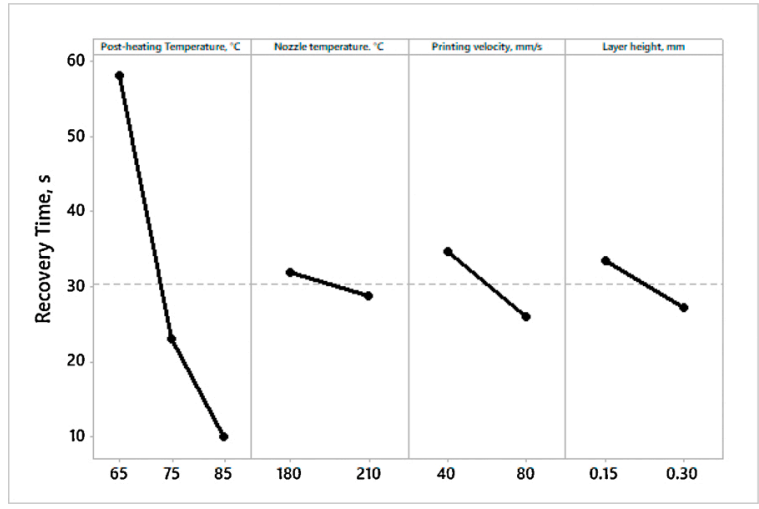

Fig. 9. Main effects plot of the recovery time vs. operating parameters.

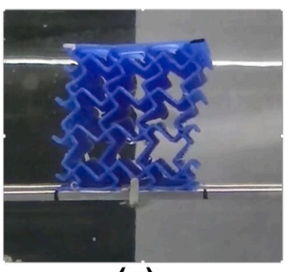

(c)

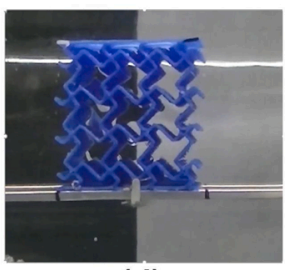

(d)

Fig. 8. Recovery steps of the sample: (a) immediately after programming; (b), (c) after a while; (d) at the end of the recovery. 


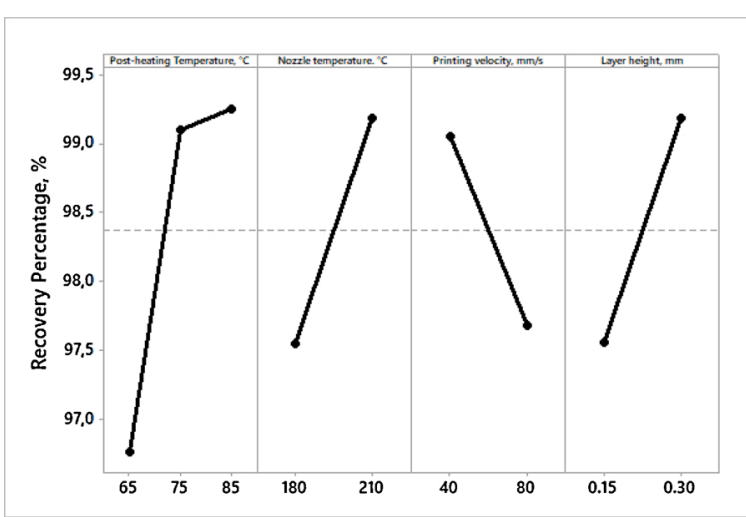

Fig. 10. Main effects plot of the recovery percentage vs. operating parameters.

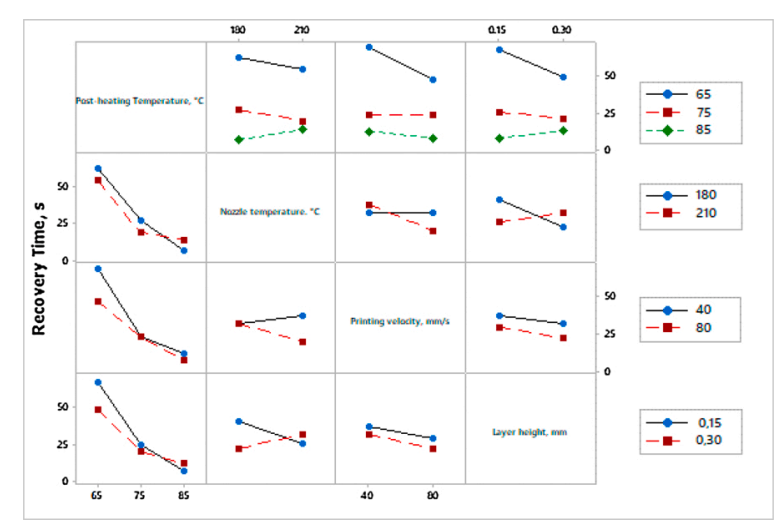

Fig. 11. Interactions plot of the recovery time vs. operating parameters.

decreases. At the same time, the material is drawn during the printing process by the fast-moving head, this being the reason of the onset of massive planar stresses inside the polymeric material. Such stresses can cause a decrease in recovery time but, also, a lower capability to the shape recovery of the investigated samples in agreement with [30].
Non-linear trends of the recovery time and percentage is achieved by varying the post-heating temperature together with one of the other operating parameters (nozzle temperature, printing velocity or layer height). The trends of the recovery time are non-linear and decreasing. The trends decrease when the post-heating temperature progressively increases, thus allowing faster recovery. Vice versa, the trends of the recovery percentage are non-linear and increasing for a progressively increasing post-heating temperature, which allows the samples to recover the initial shapes at a bigger extent. Therefore, the interactions plot confirms the results according to which an increase in post-heating temperature is a controlling factor for activating the PLA-based SMPs. An increase in post-heating temperature shortens the recovery times and favors a slight increase in the extent of volumetric recovery in agreement with [31]. Observing the other mini-plots in Figs. 11 and 12, some of them features curves that do not run parallel, with mutual interactions occurring among the operating parameters. The interactions seem to be stronger for the parameter nozzle temperature both with the parameter printing velocity and layer height.

This result can be probably be ascribed to the role of the nozzle temperature in the establishment of the appropriate melt pressure of the molten PLA material at the extruder nozzle. When the nozzle temperature is too low, as earlier mentioned, the material is too viscous and difficult to process. In contrast, at $210{ }^{\circ} \mathrm{C}$, the viscosity of the PLA material is much more appropriate for $3 \mathrm{D}$ printing process, thus allowing the establishment of the right melt pressure at the extruder nozzle. When this parameter is combined with the printing speed and the layer height, such effect can be emphasized or not. Specifically, when lower nozzle temperature is combined with higher printing speed, the highly viscous material has limited time to be extruded as a result of the fast printing process. This effect is deleterious on the reliability of the printing process, thus causing both low recovery time and percentage, with a strict interrelation between the two experimental factors. Similarly, when low nozzle temperature is combined with low layer height, the instability in the printing process of the PLA-based material increases, because it is difficult to reach adequate pressure of the molten material at the extruder nozzle and because the lower layer height cause the printed material to be less homogeneous and compact. For this reason, there is a concurrent effect on both the recovery time and percentage and the two experimental factors are, therefore, correlated. The other interactions are nearly negligible, with all the other trends in Figs. 11 and 12 running

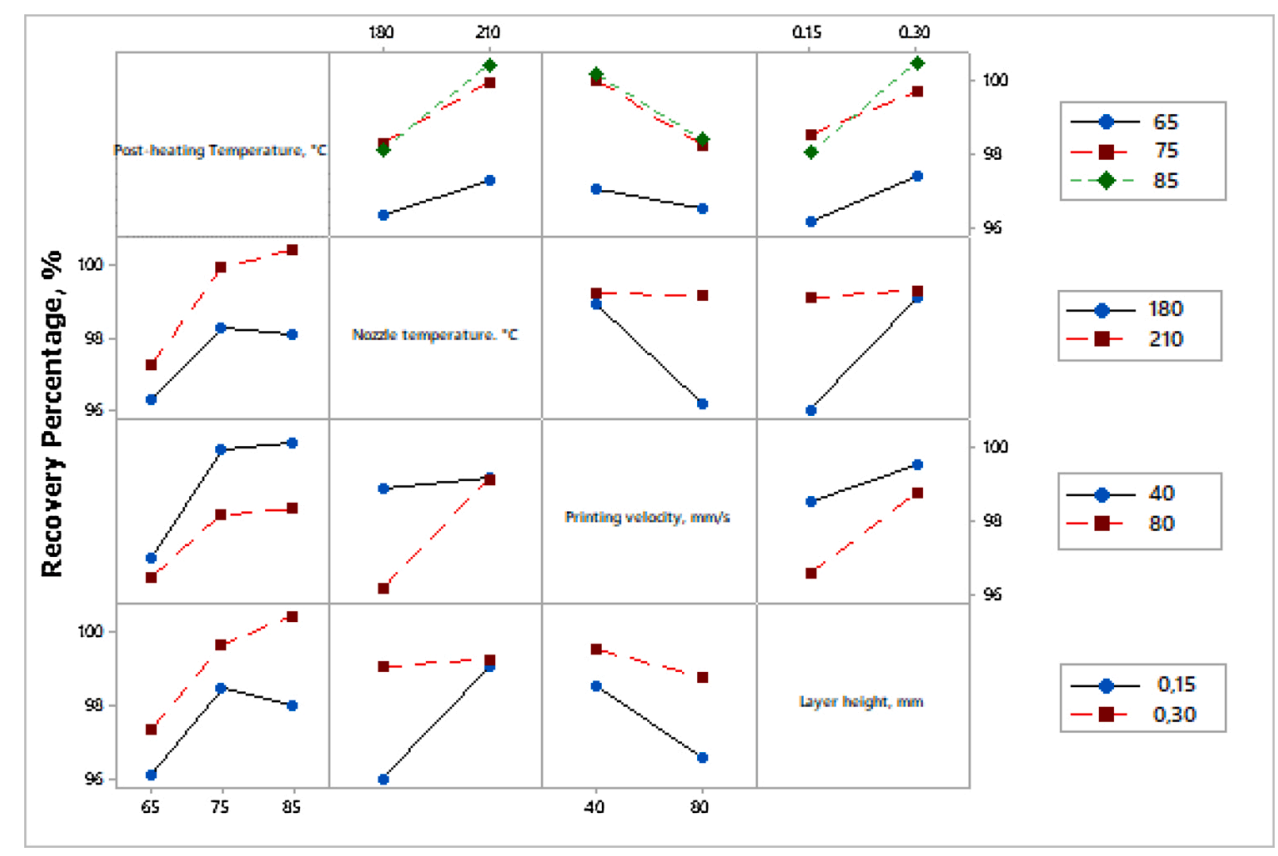

Fig. 12. Interactions plot of the recovery percentage vs. operating parameters. 
almost parallel each other.

\section{Conclusions}

In this study, PLA is used in the $4 \mathrm{D}$ printing process for the manufacturing of complex geometry components by Fused Deposition Modelling (FDM), with a potential in the manufacturing of stressabsorbers. The shape memory properties of the manufactured component have been tested varying the operational parameters of the printing process (i.e., the temperature at the nozzle, the deposition speed, the layer thickness) as well as the activation temperature that allows the recovery of the initial shape of the model. The following pointwise conclusions can be drawn:

- Programming the SMPs was performed by compressive deformation at constant deformation speed (maximum deformation was kept constant at $8 \mathrm{~mm}$ ); the trends of the compressive load by varying the nozzle temperature, the printing speed and the layer thickness show three successive peaks, which correspond to the collapse of the three layers of cellular structures of which each individual sample is composed;

- The analysis of the second peaks of the compressive load show an increase of the load by increasing the nozzle temperature from 180 to $210{ }^{\circ} \mathrm{C}$ and the layer thickness from 0.15 to $0.30 \mathrm{~mm}$. In contrast, a decreasing of the compressive load at the second peak by increasing the printing speed from 40 to $80 \mathrm{~mm} / \mathrm{s}$ is observed.

The experimental tests aimed at showing the properties of shape memory of the PLA-based printed samples have shown that PLA components have adequate shape memory, with short recovery times and high recovery rates being easily achievable. In particular, the following additional considerations can be drawn:

- Among process parameters, the activation temperature, that is, the post-heating temperature of the models is found to be the most significant parameter to trigger the recovery of the initial shape in the shortest possible time;

- An important role is also assumed by the choice of the nozzle temperature, with higher temperature allowing the setting of the right viscosity of the molten material in the extruder and the establishment of an adequate melt pressure at the extruder nozzle, that also favors both the recovery time and percentage.

In conclusion, engineered PLAs, printed with 3D technology in adequately designed cell structures, boast excellent shape recovery properties, thus being a valid candidate as an alternative and low environmental impact solution for the manufacture of energy-absorbing barriers as well as in the manufacturing of a wide variety of aeronautic components.

\section{Declaration of Competing Interest}

The authors reported no declarations of interest.

\section{Appendix A. Supplementary data}

Supplementary data associated with this article can be found, in the online version, at https://doi.org/10.1016/j.jmapro.2020.11.036.

\section{References}

[1] Kyobula M, Adedeji A, Alexander M, Saleh E, Wildman R, Ashcroft I, et al. 3D inkjet printing of tablets exploiting bespoke complex geometries for controlled and tuneable drug release. J Control Release 2017;261:207-15.
[2] Lin D, Jin S, Zhang F, Wang C, Wang Y, Zhou C, Cheng G. 3d stereolithography printing of graphene oxide reinforced complex architectures. Nanotechnology 2015;26(43):434003 (9pp).

[3] Liu L, Wang X, Guo P. Influences of three-dimensional printing to product innovation design thinking. In: MATEC Web of Conferences. 100; 2017.

[4] Fullerton J, Frodsham G, Day R. 3D printing for the many, not the few. Nat Biotechnol 2014;32(1):1086-7.

[5] Lewis J. 3D tissue printing. Front Bioeng Biotechnol 2016;4.

[6] Matos F, Godina R, Jacinto C, Carvalho H, Ribeiro I, Peças P. Additive manufacturing: exploring the social changes and impacts. Sustainability 2019;11 (14):3757.

[7] Marak Z, Tiwari A, Tiwari S. Adoption of 3D printing technology: an innovation diffusion theory perspective. Int J Innov 2019;7(1):87-103.

[8] Mankar S, Kale C, Kanchan J. 3D printing technology-A computer aided Design-A review. Res J Sci Technol 2019;11(3):217.

[9] Rayate A, Jain P. A review on 4D printing material composites and their applications. Materialstoday: Proc 2018;5(9):20474-84.

[10] Kaynak C, Varsavas S. Performance comparison of the 3D-printed and injectionmolded PLA and its elastomer blend and fiber composites. J Thermoplast Compos Mater 2019;32(4):501-20.

[11] Griffin J. The state of creativity: the future of 3D printing, 4D printing and augmented reality. Northampton, Massachusetts, USA: Edward Elgar Publishing; 2019.

[12] Behl M, Lendlein A. Shape-memory polymers. Mater Today 2007;10(4):20-8.

[13] Zhang Q, Yan D, Hu G. Pattern transformation of heat-shrinkable polymer by threedimensional (3D) printing technique. Sci Rep 2015;5:8936.

[14] Bodaghi M, Noroozi R, Zolfagharian A, Fotouhi M, Norouzi S. 4D printing selfmorphing structures. Materials 2019;12(8):1353-64.

[15] Liu Z, Wang Y, Wu B, Cui C, Guo Y, Yan C. A critical review of fused deposition modeling 3D printing technology in manufacturing polylactic acid parts. Int J Adv Manuf Technol 2019;102(9):2877-99.

[16] Liu CQ. Review of progress in shape-memory polymers. J Mater Chem 2007;17 (16):1543-58.

[17] Leist S, Gao D, Chiou R, Zhou J. Investigating the shape memory properties of 4D printed polylactic acid (PLA) and the concept of 4D printing onto nylon fabrics for the creation of smart textiles. Virtual Phys Prototyp 2017;12(4):290-300.

[18] Kumar R, Singh R, Farina I. On the 3D printing of recycled ABS, PLA and HIPS thermoplastics for structural applications. Psu Res Rev 2018;2(2):115-37.

[19] Dogan S, Boyacioglu S, Kodal M, Gokce O, Ozkoc G. Thermally induced shape memory behavior, enzymatic degradation and biocompatibility of PLA/TPU blends: "Effects of compatibilization". J Mech Behav Biomed Mater 2017;71: 349-61.

[20] Liu Y, Zhang W, Zhang F, Lan X, Leng J, Liu S, Jia X, Cotton C, Sun B, Gu B, Chou TW. Shape memory behavior and recovery force of $4 \mathrm{D}$ printed laminated Miura-origami structures subjected to compressive loading. Compos Part B Eng 2018;153:233-42.

[21] Barletta M, Puopolo M. Thermo-mechanical properties of injection molded components manufactured by engineered biodegradable blends. J Polym Environ 2019;27(10):2105-18.

[22] Malekzadeh K, Khalili M, Jafari A, Mittal R. Dynamic response of in-plane prestressed sandwich panels with a viscoelastic flexible core and different boundary conditions. J Compos Mater 2006;40(16):1449-69.

[23] He Q, Feng J, Chen Y, Zhou H. Mechanical properties of spider-web hierarchical honeycombs subjected to out-of-plane impact loading. J Sandw Struct Mater 2020; 22(3):771-96.

[24] Ligon S, Liska R, Stampfl J, Gurr M, Mülhaupt R. Polymers for 3D printing and customized additive manufacturing. Chem Rev 2017;117(15):10212-90.

[25] Gordeev E, Galushko A, Ananikov V, McAlpine M. Improvement of quality of 3D printed objects by elimination of microscopic structural defects in fused deposition modeling. PLoS One 2018;13(6):e0198370.

[26] Zhang H, Chen Z, Zheng Z, Zhu X, Wang H. Shape memory polymer hybrids of SBS/ dl-PLA and their shape memory effects. Mater Chem Phys 2013;137(3):750-5.

[27] Radjabian M, Kish M, Mohammadi N. Structure-property relationship for poly (lactic acid) (PLA) filaments: physical, thermomechanical and shape memory characterization. J Polym Res 2012;19(6):1-10.

[28] Lai S-M, Lan Y-C. Shape memory properties of melt-blended polylactic acid (PLA)/ thermoplastic polyurethane (TPU) bio-based blends. J Polym Res 2013;20(5):1-8.

[29] Navarro-Baena I, Sessini V, Dominici F, Torre L, Kenny J, Peponi L. Design of biodegradable blends based on PLA and PCL: from morphological, thermal and mechanical studies to shape memory behavior. Polym Degrad Stab 2016;132: 97-108.

[30] Casavola C, Cazzato A, Moramarco V, Pappalettera G. Residual stress measurement in fused deposition modelling parts. Polym Test 2017;58:249-55.

[31] Paakinaho K, Heino H, Pelto M, Hannula M, Törmälä P, Kellomäki M. Programmed water-induced shape-memory of bioabsorbable poly(d,l -lactide): activation and properties in physiological temperature. J Mater Sci Mater Med 2012;23(3): 613-21. 